\title{
Dentists should play a greater role in detecting diabetes and heart disease
}

Patient health could be significantly improved if dentists played a greater role in detecting health conditions such as diabetes and cardiovascular disease, it has been suggested.

The Faculty of Dental Surgery (FDS) at the Royal College of Surgeons, has suggested a boosted role for dentists in identifying certain health conditions beyond the typical oral health field.

In a Position Statement on oral health and general health ${ }^{1}$ published on 25 April 2019, the FDS said the recently published NHS Long Term Plan ${ }^{2}$ emphasised the importance of preventing ill health and included commitments around improving the oral health of children, older people, and those with learning disabilities. The government's green paper on prevention is also due to be published later this year.

In this context, there was an opportunity to reflect on what dentistry was for and how oral health professionals could provide the best possible care for their patients.

The position statement details evidence of the link between oral and general health such as a series of rapid research reviews published in 2017 which were supported by the FDS and other leading dental organisations.

These reviews, ${ }^{3}$ published in the $B D J$, considered the links between oral health and a number of broader health conditions.

One rapid research review $w^{4}$ published in the $B D J$ highlighted that current evidence indicated a number of associations between diabetes and oral diseases, and that diabetes was a recognised risk factor for gum disease.

The FDS' position statement suggests that dentists could play a greater role in supporting patients' general health, both by helping to diagnose certain wider health problems and by providing preventative health advice.

In order to maximise the impact that dentists and oral health professionals could have to support patients' general health, the FDS recommends that:

- Oral health be included in the government's upcoming green paper on prevention

- National and local public health campaigns should always utilise dentists in the delivery of health and lifestyle advice while awareness should be raised amongst the general public about the links between oral and general health

- Initiatives to diagnose diabetes and cardiovascular disease, as well as other conditions such as child obesity and eating disorders, should engage dentists and oral health professionals wherever possible

- All healthcare professionals should cover the links between oral health and general health as part of their initial training and continuing professional development

- Concerted action is needed to improve oral care and access to dental services

\begin{abstract}
for older people, including those living
\end{abstract} in care homes.

Professor Michael Escudier, Dean of the FDS, said: 'Good oral health is essential for our overall wellbeing. In recent years there has been increasing evidence of the link between oral health and general health. Dentists and other members of the oral healthcare team always inspect a patient's mouth in the course of treatment.

'This provides them with an opportunity to monitor, on an ongoing basis, how their patient's health is changing. While checking a patient's oral health, they can look for relevant signs of other conditions - chronic gum disease can be an indicator of diabetes and cardiovascular disease, for example. They can also offer advice on what dietary and lifestyle changes patients could make to improve their overall health, which can also help to prevent conditions such as obesity and oral cancer.'

\section{References}

1. Faculty of Dental Surgery, Royal College of Surgeons Position Statement: Oral health and general health. 2019. Available at https://www.rcseng.ac.uk/ dental-faculties/fds/faculty/government-relations-and-consultation/fds-reports/ (accessed April 2019)

2. Department of Health \& Social Care. The NHS Long Term Plan. 2019. Available at https://www.longtermplan.nhs.uk/ (accessed April 2019).

3. Klass C, Wanyonyi K, White S, Walmsley A D, Hunt N Gallagher J E. A recipe for future research. Br Dent J 2017; 222: 321

4. D'Aiuto F, Gable D, Syed Z et al. Evidence summary: The relationship between oral diseases and diabetes. Br Dent J 2017; 222: 944-948.

\section{BDI RESEARCH INSIGHTS BDJ FOR ANOTHER VIEW}

- Research insights from across the BDJ Portfolio

- Providing busy readers with valuable context including article summaries and expert commentary

Catch up here: http://bit.ly/BDJResearchlnsights

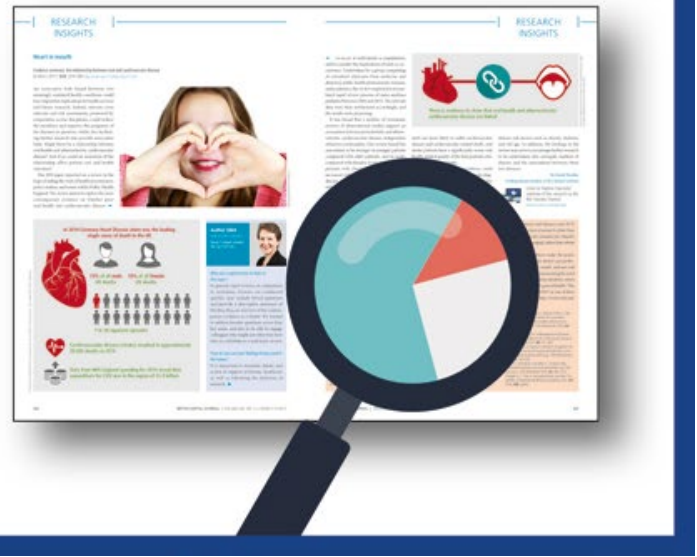

